\title{
Identification of downstream target genes regulated by CX43 in hepatocellular carcinoma
}

\author{
Z. S. WANG ${ }^{1, *}$, Z. TAN ${ }^{1, *}$, Z. H. WU ${ }^{1}$, S. X. ZHAN ${ }^{1}$, W. D. GUO ${ }^{1}$, S. G. LIU ${ }^{2, *}$, L. ZHANG ${ }^{2, *}$ \\ ${ }^{1}$ Department of Hepatobiliary and Pancreatic Surgery, The Affiliated Hospital of Qingdao University, Qingdao, China; ${ }^{2}$ Prenatal Diagnosis \\ Center, The Affiliated Hospital of Qingdao University, Qingdao, China \\ *Correspondence: liushiguo2002@126.com; 0130aici@163.com \\ ${ }^{\#}$ Contributed equally to this work.
}

Received December 25, 2018 / Accepted March 20, 2019

\begin{abstract}
The aim of study was to identify the downstream target genes of CX43 by Human Transcriptome Array. Therefore, a gene microarray was generated consisting of CX43-overexpressed hepatocellular carcinoma (HCC) cells, transfected with the constructed plasmid and negative controls, to identify candidate genes. Integrated bioinformatic analysis was used to clarify biological functions of the identified genes, including Gene Ontology (GO), Kyoto Encyclopedia of Genes and Genomes (KEGG) pathway, protein-protein interaction network and survival analysis. The candidate genes were further validated by qRT-PCR in liver cancer tissues and CX43-silenced HCC cells. We have found the mRNA and protein levels of CX43 significantly upregulated in HCC cells transfected with CX43 constructed plasmid. We identified 928 differentially expressed genes including 394 upregulated and 534 downregulated genes, enriched in the cancer related functions and pathways by GO and KEGG pathway analysis. The protein-protein interaction network revealed 9 hub genes in this study. Statistical analysis indicated that upregulation of RALA and SRC was associated with poor prognosis in liver cancer. The differential expressions of 2 candidate genes were further validated in HCC cells and tissues. In conclusion, protein-coding genes RALA and SRC could be target genes of CX43 and therapeutic targets for hepatocellular carcinoma.
\end{abstract}

Key words: hepatocellular carcinoma, connexin 43, human transcriptome array

Hepatocellular carcinoma (HCC) is one of the most common lethal cancers worldwide [1]. It is acknowledged that the main carcinogenic factors of HCC are hepatitis $B$ virus (HBV), hepatitis $\mathrm{C}$ virus (HCV), alcohol and non-alcoholic steatohepatitis. Although surgical techniques have been constantly refining over recent years, there is a high tendency for recurrence and distant metastasis of HCC after radical resection. Previous research has delineated that 5 -year survival rate of HCC patients who underwent radical resection can exceed 50\% [2]. However, the recurrence rate of HCC is still as high as $70 \%$ after 5 years, which rises an urgent need for new therapeutic strategy.

Connexins (CX) are one of the principal elements of gap junction access to intercellular communication and homeostasis. In chordates, CX can constitute two structures in cell: hemichannels (HCs) and gap junction channels (GJCs). They could be as a media between intracellular and extracellular or communicate adjacent cells by cytoplasm, respectively [3]. Indeed, there is growing evidence highlighting the important role of CX43 in the tumorigenicity and cancer progression.
Connexins can regulate cell specific functions, such as cell apoptosis and differentiation, the abnormity of which can lead to physical abnormalities such as cardiac failure and cancer [4]. The downregulation of CX43 in HCC is related to cancer metastasis, angiogenesis and epithelial to mesenchymal transition (EMT) [5]. Previously, we have proved that the expression of CX43 in HCC tissues was lower than in paracancerous tissue and cirrhosis tissue by immunohistochemistry and RT-PCR. Additionally, we also revealed that CX43 could be a predictor in HCC of lower early recurrence rates and indicated a novel link between CX43 expression and prognosis in patients with lower AFP level [6]. However, the downstream mechanism of CX43 on HCC progression is largely unknown.

Human Transcriptome Array technology is an effective high-throughput sequencing technique to measure gene expression profiles [7]. Moreover, bioinformatics methods were widely utilized to identify novel biomarkers and therapeutic targets for human diseases [8,9]. Recently, Human Transcriptome Array has been broadly used in cancer 
research accompanied with bioinformatics methods [10]. In this study, we applied the Human Transcriptome Array to identify the target genes in downstream mechanisms governed by CX43. We generated Transcriptome Array data from CX43-overexpressed HCC cells and then identified some candidates. Bioinformatics methods were utilized to identify differentially expressed genes (DEGs) and conducted of function and pathway enrichment analyses. Moreover, through protein-protein interaction (PPI) network and survival analysis, we validated 2 hub genes (RALA and SRC) genes and confirmed them in collected liver cancer tissues as well as CX43-silenced HCC cells. Overall, this study identifies that RALA and SRC have a correlation with HCC and could be a new signature as target genes of CX43.

\section{Materials and methods}

Cell cultures. Human liver cancer cell line SMMC-7221 was obtained from Cell Bank (Chinese Academy of Sciences, Shanghai). The cells were maintained in the DMEM (Hyclone, Shanghai, China) supplemented with $10 \%$ fetal bovine serum $1 \%$ penicillin-streptomycin at $37^{\circ} \mathrm{C}$ in a $5 \% \mathrm{CO}_{2}$ atmosphere.

Construction of over-expressed plasmid and cell transfection. The CX43 overexpressing plasmid was constructed as follows: full-length CX43 complementary DNA was inserted into the Xho I and BamH I site in sense direction in the eukaryotic expression vector pcDNA3.1(+) (Genechem, China). The plasmid DNA was extracted from cultured competent cells by overnight shaking $(200 \mathrm{rpm})$ at $37^{\circ} \mathrm{C}$ using EndoFree Mini Plasmid Kit II (TianGen Biotech, Beijing, China) according to the manufacturer's instructions. Transfection were performed using Lipofectamine ${ }^{\mathrm{TM}} 3000$ (Invitrogen, Carlsbad, USA) according to the manufacturer's protocol and identified by qRT-PCR and western blot, respectively.

RNA extraction, cDNA synthesis and real-time quantitative polymerase chain reaction (qRT-PCR). Total RNA of SMMC-7721 cells was isolated after 24 hours of transfection by Trizol (Invitrogen, Carlsbad, U.S.A.) following the manufacturer's instructions. $1 \mu \mathrm{g}$ of total RNA was used for cDNA synthesis with TransScript One-Step gDNA Removal and cDNA Synthesis SuperMix (Transgen, Beijing, China). A qRT-PCR kit (Transgen, Beijing, China), the corresponding gene-specific primers and a TIB8600 Real-Time PCR System (Triplex International Biosciences, China) were used for amplifying cDNA. Primer syntheses were completed by Sangon Biotech, China. Primers sequences for CX43 forward: 5'-TTCTTGCTGATCCAGTGGTACATC-3', reverse: 5'-CAAGGACACCACCAGCATGA-3'; for GAPDH forward: 5'-CTGACTTCAACAGCGACACC-3', reverse: 5'-TGCTGTAGCCAAATTCGTTGT-3'. The reaction conditions of q-RT PCR were used as follows: pre-denaturation at $94^{\circ} \mathrm{C}$ for 30 seconds; the amplification procedure of $95^{\circ} \mathrm{C}$ 5 seconds, $60^{\circ} \mathrm{C} 15$ seconds and $72^{\circ} \mathrm{C} 10$ seconds operated 40 cycles; $\mathrm{CQ}$ value calculated and repeated three times for each sample. After the PCR reaction was finished, the product melting curve was analyzed to confirm that it is single and the data could be used. Data were standardized with GAPDH as the internal reference and the expression levels of target genes were analyzed using comparative threshold cycle method $\left(2^{-\Delta \Delta C T}\right)$. Results are means \pm SD from 3 independent experiments performed in duplicate.

Western blotting. After 48 hours of SMMC-7721 cells transfection, cells $\left(1 \times 10^{6}\right.$ cells) were washed with PBS two times, and then lysed by $150 \mu$ RIPA Lysis Buffer with $1 \%$ phenylmethanesulfonyl fluoride (PMSF) mixture for 30 minutes. Afterwards, the lysates were centrifuged at $4{ }^{\circ} \mathrm{C}$ with $12000 \times \mathrm{g}$ for 15 minutes and the supernatant was collected. Enhanced BCA protein assay kit was used to detected protein concentration (Beyotime, Shanghai, China). $25 \mu \mathrm{g}$ of standard protein solution was diluted to $20 \mu \mathrm{l}$ with PBS and $5 \mu \mathrm{l}$ protein buffer was added, followed by the incubation in a OSE-DB-01 metal bath (TIANGEN Biotech, Beijing, China) for 10 minutes at $95^{\circ} \mathrm{C}$. The obtained protein samples were added to $10 \%$ SDS-PAGE separation gel to separate the total protein and later transferred onto the polyvinylidene fluoride (PVDF) membrane. Then the membrane was blocked with $5 \%$ skimmed milk at room temperature for 1 hour followed by incubation overnight at $4{ }^{\circ} \mathrm{C}$ with antibody at 1:300 dilution for GJA1 (BA1727, Boster, China) and 1:1000 for GAPDH (AB9483, Abcam, USA). The secondary antibody coupled to the appropriate horseradish peroxidase (HRP) with the dilution 1:5000 was used for incubation at room temperature for 1 hour. The protein bands were detected by enhanced chemiluminescence (Thermo Fisher Scientific, USA). Results are means \pm SD from 3 independent experiments performed in duplicate.

SiRNA transfection. To silence the CX43 expression, we transfected small interfering RNAs into SMMC-7721 cells. The target sequences of siRNAs were as follows: CX43 siRNA\# 1 5'-CCGCAAUUACAACAAGCAATT-3'; CX43 siRNA\#2 5'-CCUGGCUCAUGUGUUCUAUTT-3'; CX43 siRNA\#3 5'-CCCUGGCCUUGAAUAUCAUTT-3'; negative control 5'-UUCUCCGAACGUGUCACGUTT-3'. siRNAs were transfected into SMMC-7721 cells using Lipofectamin ${ }^{\mathrm{Tm}}$ 3000 referring to manufacturer's instructions. Total RNA and protein was extracted 24 and 48 hours post-transfection, then qRT-PCR and western blotting were used to detect the knockdown efficiency.

Tissue samples. We collected eighty HCC tissues samples including forty tumors and forty paired adjacent noncancerous tissue ( $3.0 \mathrm{~cm}$ away from the margin of cancer tissue) from Hepatological Surgery Department of Affiliated Hospital of Qingdao University. All collected cases were histopathologically confirmed as HCC. The collected HCC tissues and paired adjacent noncancerous tissues were harvested and immediately immersed in the RNA stabilization reagent (Kangwei, Beijing, China) and stored at $-80^{\circ} \mathrm{C}$ until use. The project was authorized by the Ethics Committee of the Affiliated Hospital of Qingdao University. Written informed consent documents was signed by the patients involved in the study. 
Microarray analysis. Microarray profiling was performed using Clariom $^{\text {Tm }}$ D Assay (No. 902923, Affmetrix, Thrmo Fisher). Briefly, kit generates amplified and biotinylated sense-strand DNA targets generated from $100 \mathrm{ng}$ of total RNA from each of six samples. The RNA was then reverse transcribed to synthesize complementary RNA (cRNA) using GeneChip ${ }^{\text {mu }}$ WT Plus kit (Catalogue No. 902280; Applied Biosystems). Bi-weight in bioconductor was needed to calculate differential gene expressions in two groups and the Benjamini-Hochberg test was applied to evaluate the significance of differential gene expression. The p-value was fixed by the false discovery rate (FDR). We confined the gene as a differential expressed gene when FDR $<0.01$ and the absolute values of the fold change was greater than 1.5.

KEGG and GO enrichment analyses for DEGs. To get a deeper understanding of genes and proteins for target genes, we took advantage of a free online tool, DAVID, the Database for Annotation, Visualization and Integrated Discovery (http://david.ncifcrf.gov). We performed GO (Gene Ontology) and KEGG (Kyoto Encyclopedia of Genes and Genomes) for differentially expressed genes [11, 12]. A p-value $<0.05$ was considered statistically significant.

PPI network construction. The PPI network was constructed with the STRING, Search Tool for the Retrieval of Interacting Genes (http://string-db.org), online database. In our study, it suggested a statistically significant when the interaction with a combined score was $>0.4$. In addition, to visualize molecular interaction network, we utilized Cytoscape (www.cytoscape.org), a free bioinformatics platform [13].

Survival analysis. We utilized Kaplan-Meier survival and Cox regression analyses to recognize the prognostic value of the target genes. GEPIA (http://gepia.cancer-pku.cn) and Kaplan-Meier Plotter database were chosen to carry out the statistical analyses [14].

Statistical analysis. All statistical analyses were performed using R software version 3.3 and GraphPad Prism 6 software (GraphPad Software, Inc. La Jolla, USA). Receiver operating characteristic (ROC) curves and logistic regression analysis were utilized to analyze specificity, sensitivity, and area under the curve (AUC) with $95 \%$ confidence intervals (CIs). Statistical significance was examined by Student's $t$ test and $p<0.05$ or $\mathrm{p}<0.01$ was statistically significant.

\section{Results}

Verification of CX43 overexpression. To explore the target genes regulated by CX43, we constructed CX43 overexpression plasmid to overexpress CX43 in SMMC-7221. The expression of CX43 level was confirmed by qRT-PCR and western blot (Figure 1).

Identification and analysis of differentially expressed genes. The heat map demonstrated the variation among mRNA expression between two groups. The top DEGs were exhibited in a heat map (Figure 2). In total, 928 differentially expressed genes were identified including 394 upregulated and 534 downregulated genes. KEGG analysis revealed that the altered genes were concentrated on cancer pathways, such as calcium, FC gamma $\mathrm{R}$ and other cancer-related pathways (Figure 3A). In addition, GO annotation demonstrated that the DEGs were related to signal receptors, signal transducer, regulation of response to external and intrinsic component of plasma (Figure 3B).

Hub gene analysis. A PPI network was constructed for all 928 differentially expressed genes using the STRING database in the Cytoscape software. Hub gene were filtered if the adjacent p-value was $<0.05$. Consequently, CD69 (Cluster of Differentiation 69), IL6 (Interleukin 6), SOD2 (Superoxide dismutase 2, mitochondrial), ENO1 (Enolase 1), PRKCG (Protein kinase $\mathrm{C}$ gamma type), EXOC4 (Exocyst complex component 4), RALA (Ras-related protein Ral-A), SRC (SRC

A

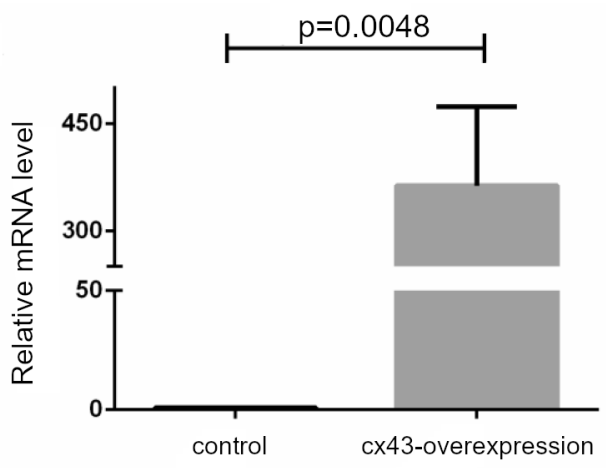

B

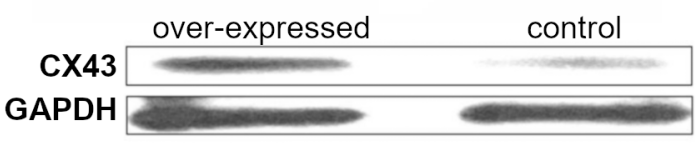

C

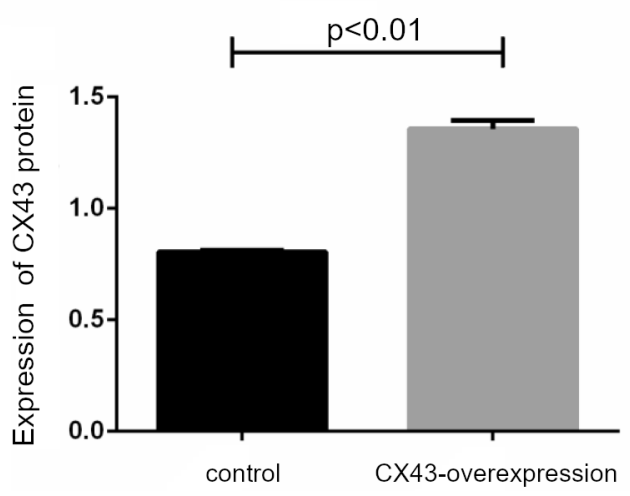

Figure 1. Overexpression of CX43 in SMMC-7721 cell line was confirmed by $q$ RT-PCR $(\mathrm{A})$ and western blot $(\mathrm{B}, \mathrm{C})$. Results are presented as mean \pm $\mathrm{SD}\left(\mathrm{n}=3\right.$ in each group, $\left.{ }^{*} \mathrm{p}<0.05\right)$. 


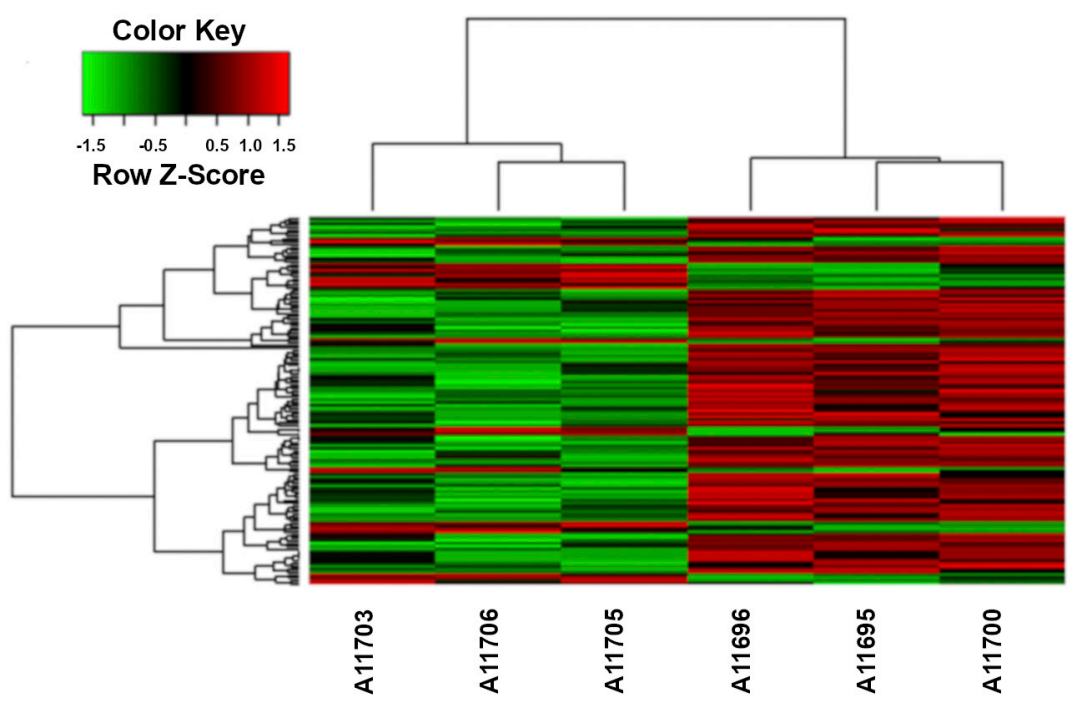

Figure 2. A heat map of differentially expressed genes analysis between CX43-overexpressed and control: 394 upregulated and 534 downregulated genes. Red means upregulation; green means downregulation.

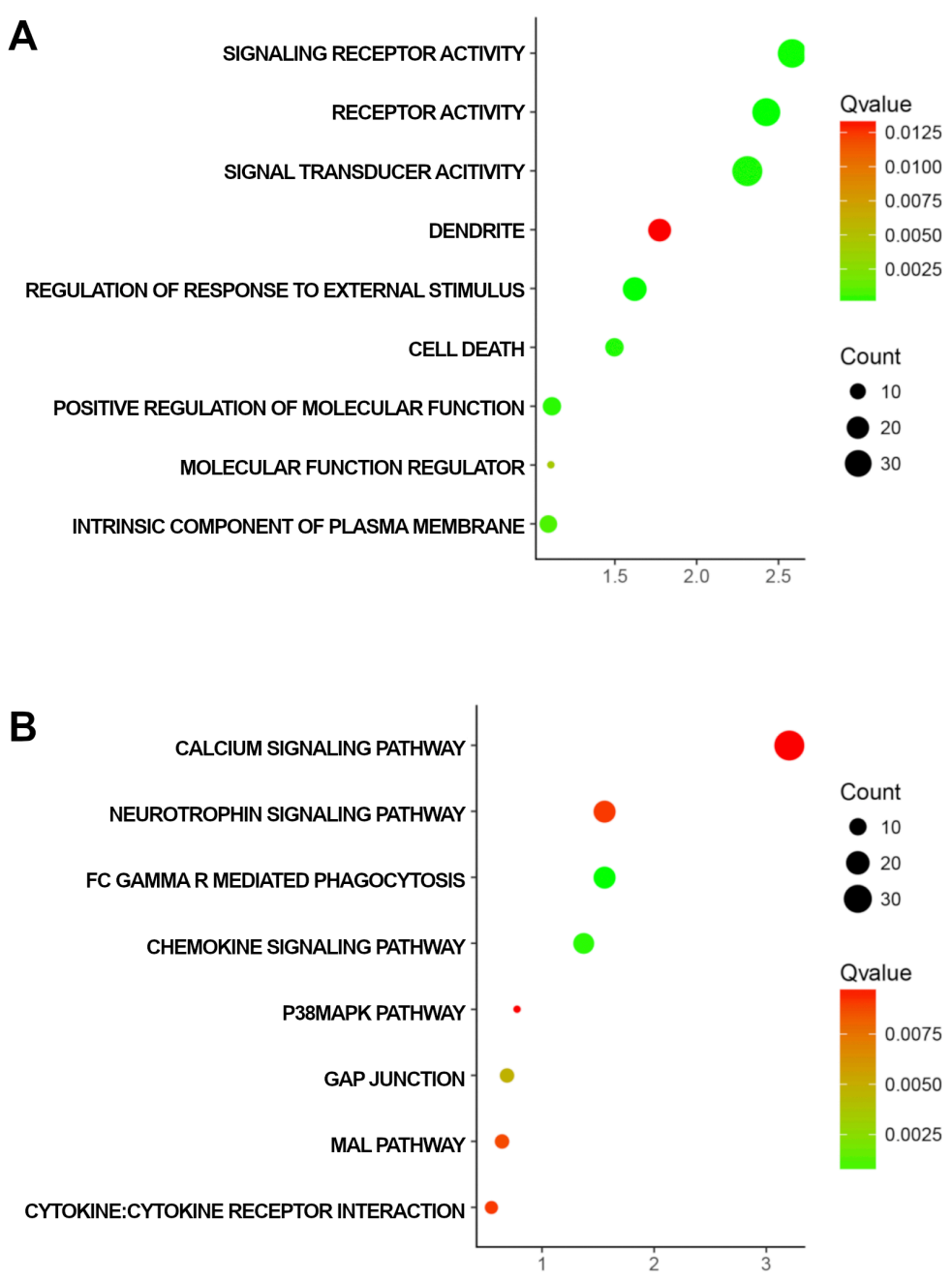

Figure 3. GO (A) and KEGG pathways (B) in differentially expressed genes. 
Proto-Oncogene) and ANKRD36 (Ankyrin Repeat Domain 36) were the top nine genes with highest degree (Figure 4).

Survival analysis. We further constructed Kaplan-Meier curve and Cox regression for the top nine genes by utilized the Kaplan-Meier-plotter and TCGA databases, and the results showed that CD69, ENO1, RALA and SRC were evidently associated with survival rate in liver cancer patients. In addition, we found that RALA and SRC were significantly highly expressed in liver cancer tissues by utilizing the TCGA database (Figures 5, 6).
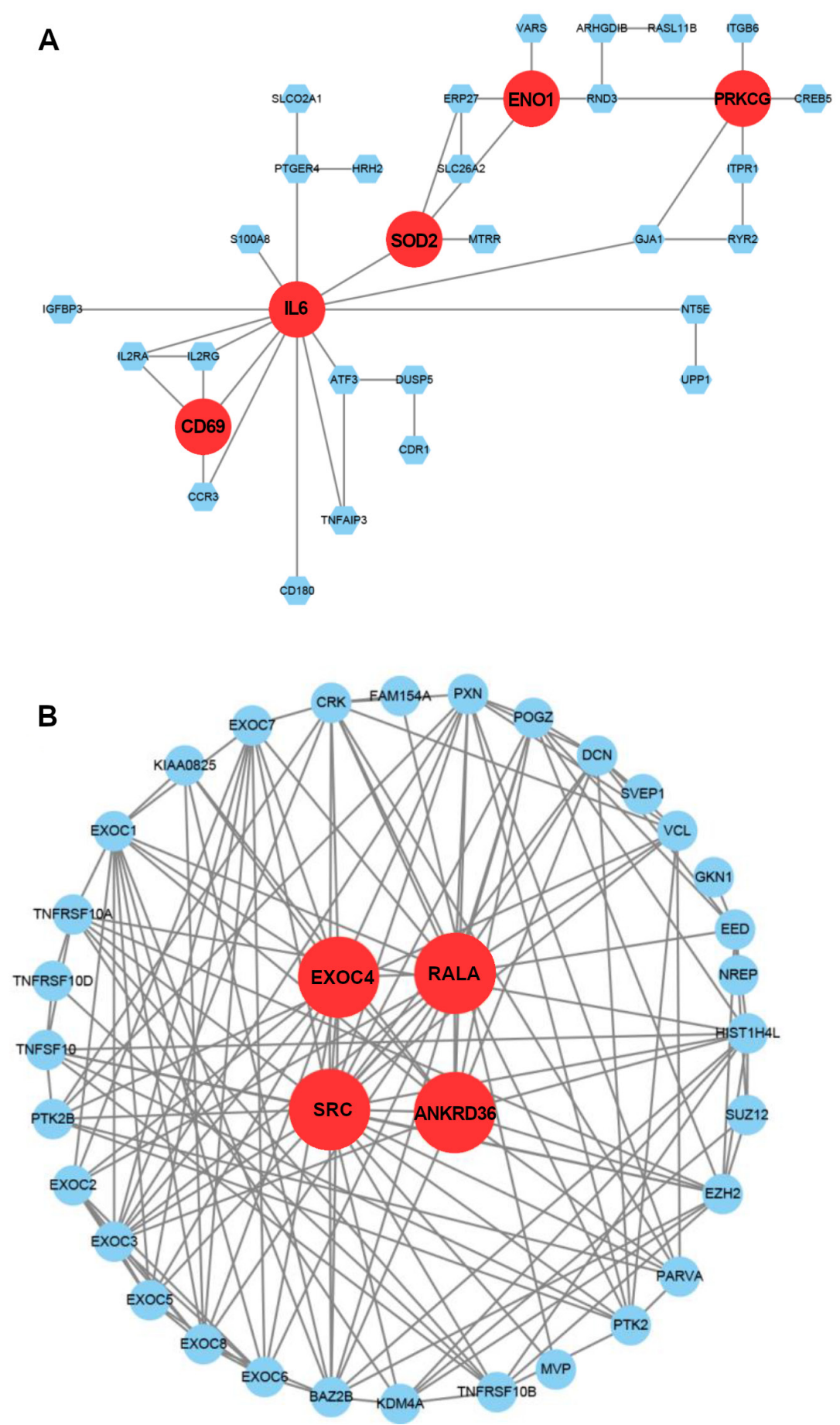

Figure 4. Protein-protein interaction network of hub genes obtained using Cytoscape software. The hub genes are CD69, IL6, SOD2, ENO1, PRKCG, EXOC4, RALA, SRC and ANKRD36. A) The upregulated protein-protein interaction network, B) The downregulated protein-protein interaction network. 

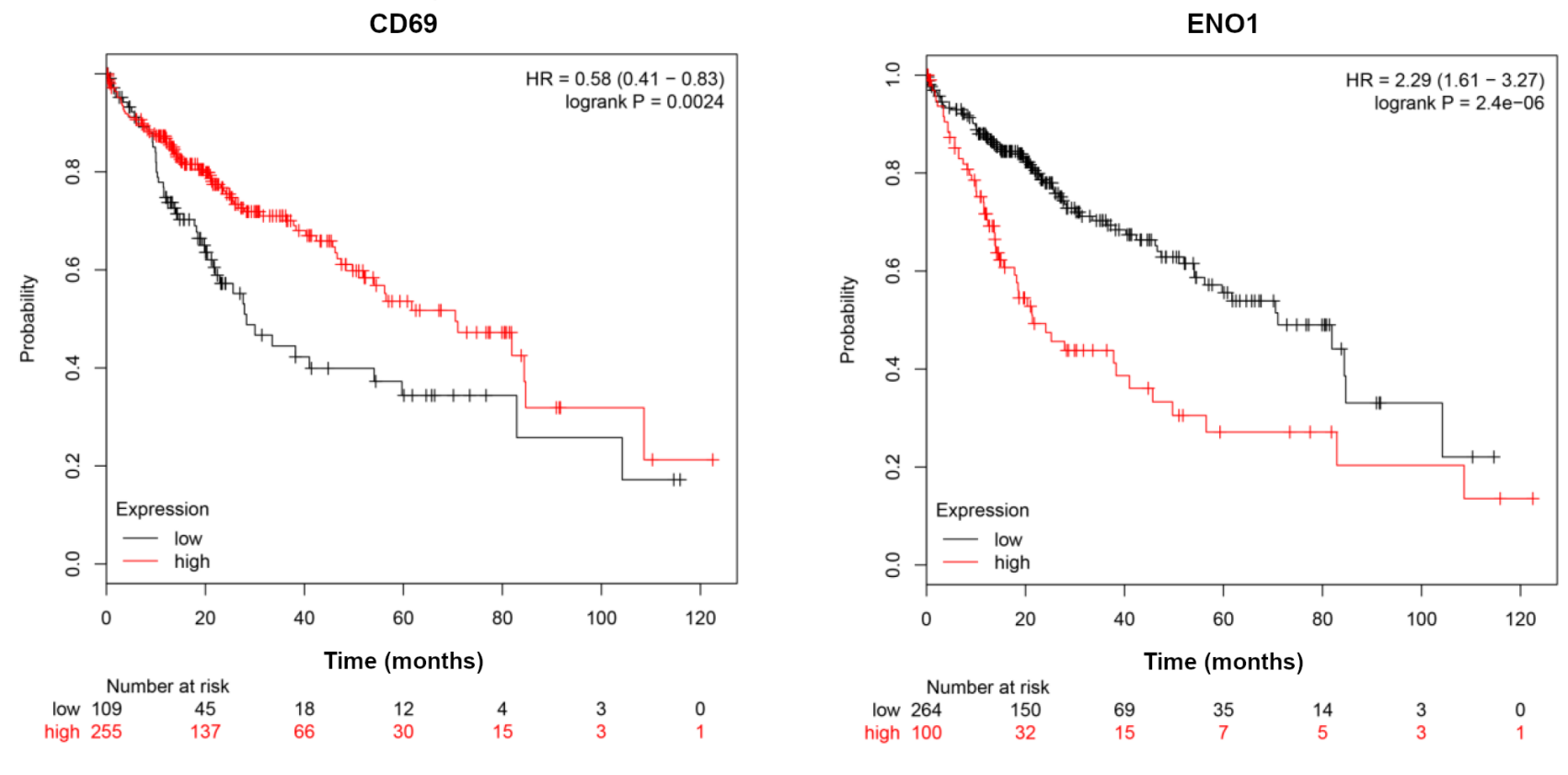

RALA

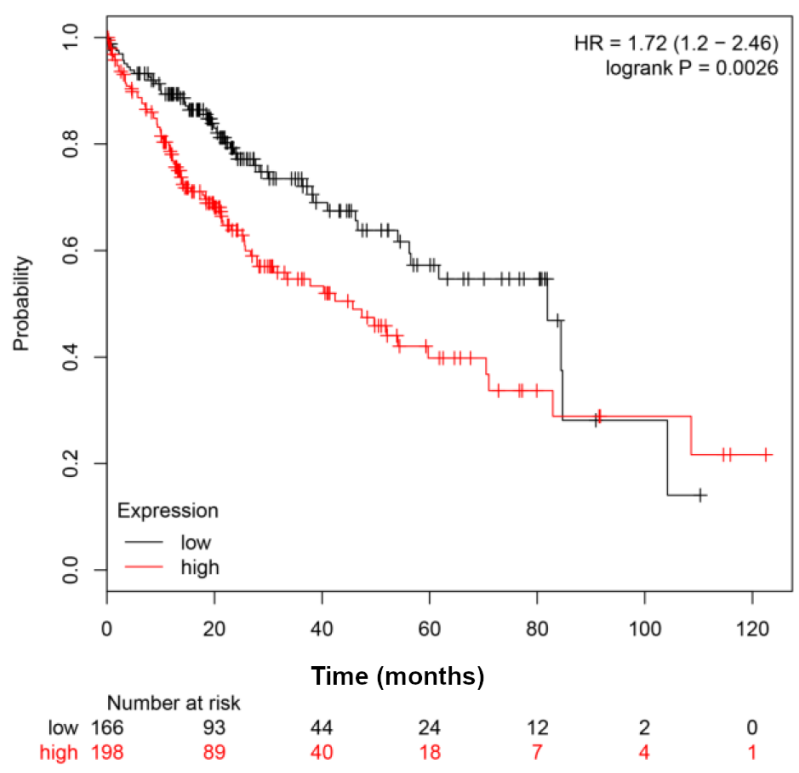

Further verification of candidate genes through qRT-PCR. To confirm our bioinformatic analyses, we utilized qRT-PCR to quantify the mRNA expression level of RALA and SRC in HCC patients ' tissues and CX43-silenced SMMC-7221 cells. Compared to normal tissues, RALA and SRC were significantly upregulated in HCC tumor samples (Figure 7). The expression of CX43 level knocked down by siRNAs was confirmed by qRT-PCR and western blot. As shown in Figure 8, RALA and SRC were significantly upregulated in CX43-silenced HCC cells.

Diagnostic value of RALA and SRC. We further assessed the diagnostic value of RALA and SRC for HCC by plotting a ROC curve (Figure 8). We found that RALA and SRC had significant diagnostic accuracy, with area under the curve (AUC) values of 0.8063 (95\% CI 0.7129-0.8996) and 0.8288 (95\% CI 0.7368-0.9207), respectively. 

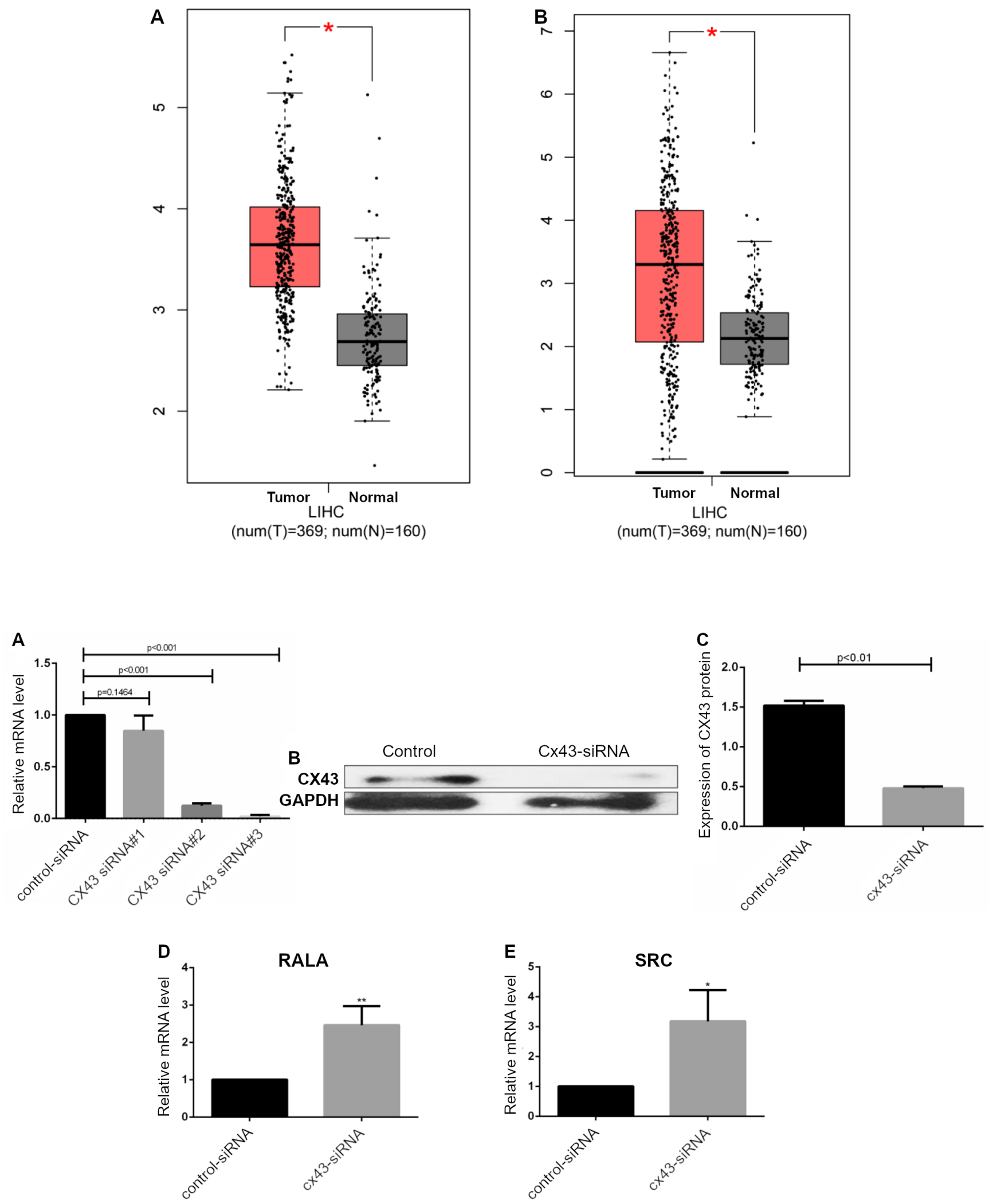
A

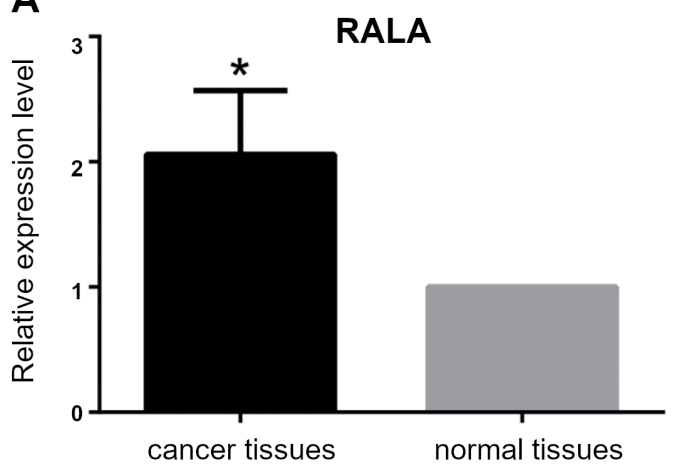

C

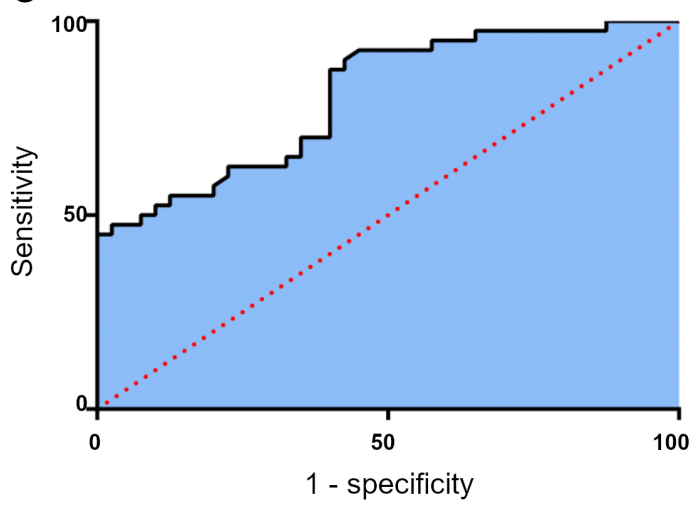

B

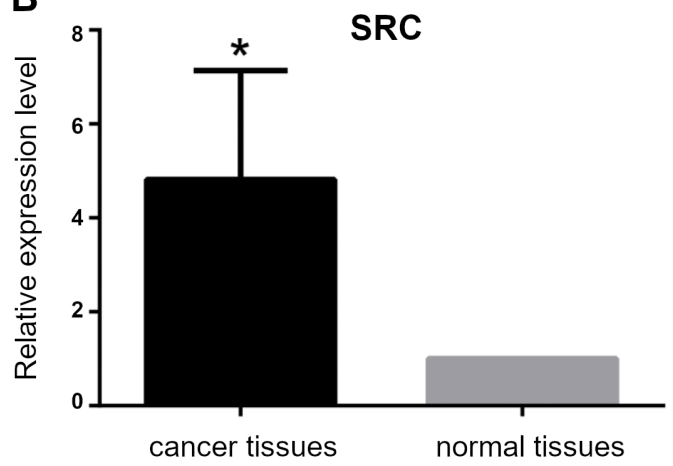

D

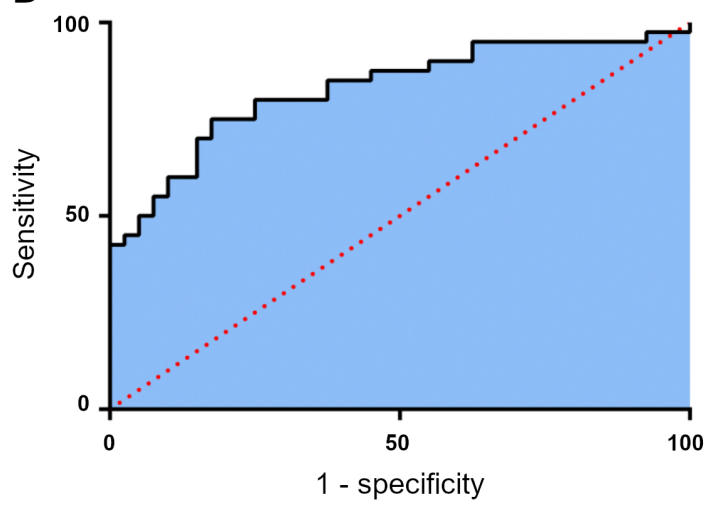

Figure 8. Verification of mRNA expression levels of RALA (A) and SRC (B) in HCC carcinoma and normal tissues with qRT-PCR. ROC analysis of RALA (C) AUC $=0.8063,95 \%$ CI 0.7129-0.8996; $p<0.001$ ) and SRC (D)AUC $=0.8288,95 \%$ CI 0.7368-0.9207; p $<0.001$ ) for discriminative ability between liver cancer cases and normal controls.

\section{Discussion}

Although CX43 has been studied in other cancers, little is known about its expression and downstream mechanism in HCC. In our previous study, we confirmed a lower expression of CX43 in $234 \mathrm{HCC}$ tissue specimens and found CX43 could delay early HCC recurrence and metastasis [6]. In this study, we identified the potential downstream targets of CX43 through the Human Transcriptome Array. Finally, 928 differentially expressed genes were identified including 394 upregulated and 534 downregulated genes. In gene ontology analysis, these DEGs illustrated an association with signal receptors and transducer and intrinsic component of plasma. Pathway analysis revealed that the candidate genes were focused on calcium signaling, Fc gamma R-mediated phagocytosis and neurotrophin signaling pathway. Subsequently, nine key hub genes (CD69, IL6, SOD2, ENO1, PRKCG, EXOC4, RALA, SRC and ANKRD36) were identified in our constructed PPI network. Further evidence of hub genes related to hepatocellular carcinoma was then provided by ONCOMINE and Kaplan-Meier-plotter databases suggesting that RALA and SRC may be responsible for liver cancer pathogenesis.
Ral (Ras like) plays a key role on pro-oncogenic signaling pathway downstream of Ras. Indeed, compared to other elements of Ral pathway, RalA was overexpressed in distinct cancer cell lines including HCC. RalA was also involved in epidermal growth factor (EGF)-mediated cell motility and tumor metastasis in human malignant tumors. In HCC, a literature supported an essential role of RalA in promoting viability and invasiveness [15]. It is possible that RalA appears to be a promising therapeutic intervention in HCC. Src, a non-receptor tyrosine kinase, could promote cell proliferation, migration and anoikis resistance in tumor cells by physical and functional interaction [16]. Previous studies showed aberrant expression of Src in various of cancer, such as colon and breast cancer $[17,18]$. Furthermore, a recent study found that the FAK/Src complex induced the epithelial mesenchymal transition and then caused solid tumor metastasis. Leca et al. reported that hepatitis B virus antigen further activated SRC/PI3K/AKT pathway through $\mathrm{PKCa} /$ RAFI pathway to promote liver cancer [19]. These results suggest that SRC could be a potential therapeutic in HCC treatment. In our study, RALA and SRC were downregulated with the overexpression of CX43 in the microarray. In addition, we found RALA and SRC had significantly higher 
expression in HCC tumor samples than normal tissues. In contrast, RALA and SRC were significantly upregulated in CX43-silenced HCC cells. These results agreed well with the findings of previous study and further indicated the $2 \mathrm{Hub}$ genes were significantly associated with CX43 expression and liver cancer survival.

To summarize, we constructed Human Transcriptome Array to identify the target genes governed by CX43 and found a set of differentially expressed protein-coding genes. Additionally, RALA and SRC were significantly associated with HCC survival. In conclusion, our study shed light on that RALA and SRC may be the target genes in the downstream mechanisms governed by CX43 and could be novel biomarkers or therapeutic targets for HCC. However, further cellular and animal experiments will be required to understand the precise mechanism of CX-43 regulating RALA and SRC in HCC development.

Acknowledgments: This work was supported by Natural Science Foundation of China (81602083).

\section{References}

[1] KAMARAJAH SK, FRANKEL TL, SONNENDAY C CHO CS, NATHAN H. Critical evaluation of the American Joint Commission on Cancer (AJCC) 8th edition staging system for patients with Hepatocellular Carcinoma (HCC): A Surveillance, Epidemiology, End Results (SEER) analysis. J Surg Oncol 2018; 117: 644-650. https://doi.org/10.1002/jso.24908

[2] AYUSO C, RIMOLA J, VILANA R, BURREL M, DARNELL A et al. Diagnosis and staging of hepatocellular carcinoma (HCC): current guidelines. Eur J Radiol 2018; 101: 72-81. https://doi.org/10.1016/j.ejrad.2018.01.025

[3] ZHAO Y, XIN Y, HE Z, HU W. Function of Connexins in the Interaction between Glial and Vascular Cells in the Central Nervous System and Related Neurological Diseases. Neural Plast 2018; 2018: 6323901. https://doi. org/10.1155/2018/6323901

[4] WEIDER K, BERGMANN M, BREHM R. Connexin 43: its regulatory role in testicular junction dynamics and spermatogenesis. Histol Histopathol 2011; 26: 1343-52. https:// doi.org/10.14670/HH-26.1343

[5] MOLICA F, FIGUEROA XF, KWAK BR, ISAKSON BE, GIBBINS JM. Connexins and Pannexins in Vascular Function and Disease. Int J Mol Sci 2018; 19. https://doi. org/10.3390/ijms19061663

[6] WANG ZS, WU LQ, YI X, GENG C, LI YJ et al. Connexin-43 can delay early recurrence and metastasis in patients with hepatitis B-related hepatocellular carcinoma and low serum alpha-fetoprotein after radical hepatectomy. BMC Cancer 2013; 13: 306. https://doi.org/10.1186/1471-2407-13-306

[7] ANSLAN S, BAHRAM M, HIIESALU I, TEDERSOO L. PipeCraft: Flexible open-source toolkit for bioinformatics analysis of custom high-throughput amplicon sequencing data. Mol Ecol Resour 2017; 17: e234-e240. https://doi. org/10.1111/1755-0998.12692
[8] ZHANG C, PENG L, ZHANG Y, LIU Z, LI W et al. The identification of key genes and pathways in hepatocellular carcinoma by bioinformatics analysis of high-throughput data. Med Oncol 2017; 34: 101. https://doi.org/10.1007/ s12032-017-0963-9

[9] THIEL WH. Galaxy Workflows for Web-based Bioinformatics Analysis of Aptamer High-throughput Sequencing Data. Mol Ther Nucleic Acids 2016; 5: e345. https://doi. org/10.1038/mtna.2016.54

[10] KABIR MF, MOHD ALI J, HAJI HASHIM O. Microarray gene expression profiling in colorectal (HCT116) and hepatocellular (HepG2) carcinoma cell lines treated with Melicope ptelefolia leaf extract reveals transcriptome profiles exhibiting anticancer activity. PeerJ 2018; 6: e5203. https://doi. org/10.7717/peerj.5203

[11] TANABE M, KANEHISA M. Using the KEGG database resource. Curr Protoc Bioinformatics 2012; Chapter 1: Unit1.12. https://doi.org/10.1002/0471250953.bi0112s38

[12] ASHBURNER M, BALL CA, BLAKE JA, BOTSTEIN D, BUTLER $\mathrm{H}$ et al. Gene ontology: tool for the unification of biology. The Gene Ontology Consortium. Nat Genet 2000; 25: 25-29. https://doi.org/10.1038/75556

[13] DEMCHAK B, HULL T, REICH M, LIEFELD T, SMOOT $\mathrm{M}$ et al. Cytoscape: the network visualization tool for $\mathrm{Ge}$ nomeSpace workflows. F1000Res 2014; 3: 151. https://doi. org/10.12688/f1000research.4492.2

[14] RHODES DR, YU J, SHANKER K, VARAMBALLY R, GHOSH D et al. ONCOMINE: a cancer microarray database and integrated data-mining platform. Neoplasia 2004; 6: $1-6$.

[15] EZZELDIN M, BORREGO-DIAZ E, TAHA M, ESFANDYARI T, WISE AL et al. RalA signaling pathway as a therapeutic target in hepatocellular carcinoma (HCC). Mol Oncol 2014; 8: 1043-1053. https://doi.org/10.1016/j. molonc.2014.03.020

[16] SURESH S, DURAKOGLUGIL D, ZHOU X, ZHU B, COMERFORD SA et al. SRC-2-mediated coactivation of anti-tumorigenic target genes suppresses MYC-induced liver cancer. PLoS Genet 2017; 13: e1006650. https://doi.org/10.1371/ journal.pgen.1006650

[17] SONG N, QU X, LIU S, ZHANG S, LIU J et al. Dual inhibition of MET and SRC kinase activity as a combined targeting strategy for colon cancer. Exp Ther Med 2017; 14: 13571366. https://doi.org/10.3892/etm.2017.4692

[18] BUKAMUR H, AQTASH O, SHAHOUB I, KAREM E, OGU I et al. Atypical rapid onset Scleroderma Renal Crisis (SRC) complicated with diffuse alveolar hemorrhage and pleuro-pericardial effusions in a patient with recently diagnosed breast cancer and a positive anti-RNA polymerase III Ab.: A case report. Respir Med Case Rep 2018; 25: 137-141. https://doi.org/10.1016/j.rmcr.2018.08.010

[19] LIU H, XU J, ZHOU L, YUN X, CHEN L et al. Hepatitis $\mathrm{B}$ virus large surface antigen promotes liver carcinogenesis by activating the Src/PI3K/Akt pathway. Cancer Res 2011; 71: 7547-7557. https://doi.org/10.1158/0008-5472.CAN-112260 